\title{
Sustained response of malignant pericardial effusion to intrapericardial bevacizumab in an advanced lung cancer patient: a case report and literature review
}

OncoTargets and Therapy

I October 2015

Number of times this article has been viewed

\section{Dawei Chen ${ }^{1,2, *}$ \\ Yan Zhang,* \\ Fang Shi ${ }^{2}$ \\ Minghuan $\mathrm{Li}^{2}$ \\ Hui Zhu ${ }^{2}$ \\ Li Kong ${ }^{2}$ \\ Jinming $\mathrm{Yu}^{2}$}

'Weifang Medical University,

Weifang, People's Republic of China; ${ }^{2}$ Department of Radiation Oncology, Shandong Cancer Hospital and Institute, Jinan, People's Republic of China

*These authors contributed equally to this work
Correspondence: Li Kong; Jinming Yu Department of Radiation Oncology, Shandong Cancer Hospital and Institute, 440 Jiyan Road, Jinan, Shandong 250I I 7 ,

People's Republic of China

Tel +86 53। 6762 6I I I/extn 697I

Fax +86 53। 87984079

Email kongli7@sina.com;

sdyujinming@।63.com

\begin{abstract}
Malignant pericardial effusion (MPCE) is a common complication of advanced malignant tumors, and interferes severely with patient prognosis and quality of life. The standard treatment for this complication is intracavitary perfusion of chemotherapeutic drugs, which is limited by unsatisfactory therapeutic effects and serious adverse events. We report a patient with MPCE who was treated with bevacizumab by pericardial perfusion, resulting in a complete response. This case supports the use of intrapericardial bevacizumab as a potential treatment for MPCE.
\end{abstract}

Keywords: bevacizumab, lung cancer, malignant pericardial effusion

\section{Introduction}

Malignant pericardial effusion (MPCE) often induces chronic pericardial tamponade and obstinate cardiac dysfunction, and thus severely diminishes patient quality of life and shortens overall survival time. ${ }^{1}$ Currently, the most common treatment for MPCE is local therapy, such as pericardial intraluminal stenting, fenestrated drainage, or pericardial perfusion of antitumor drugs, but the effectiveness of such strategies is limited. ${ }^{2}$ As reported, intrapleural and intraperitoneal perfusion of bevacizumab is effective in treating malignant pleural effusion and ascites. However, there are no data regarding intrapericardial perfusion of bevacizumab for the treatment of MPCE. We herein report a lung adenocarcinoma patient with MPCE who was treated with bevacizumab by pericardial perfusion and the resulting sustained response.

\section{Case}

A 44-year-old nonsmoking woman was admitted to our hospital in December 2014 for chest distress, asthma, and palpitation, as well as edema in her lower limbs. A computed tomography (CT) scan revealed a mass in the upper lobe of the left lung, multiple enlarged lymph nodes in the mediastinum, pleural effusion, and abundant PCE (Figure 1). Magnetic resonance imaging revealed multiple high-intensity signals in the brain. The patient had been diagnosed with adenocarcinoma of the left lung with multiple bone and brain metastases, cT4N2M1a stage IV at our hospital 17 months previously. Her EGFR-mutation status was evaluated using the amplification-refractory mutation system, which revealed an L858R point mutation in exon 21. The patient received four cycles of chemotherapy consisting of pemetrexed, cisplatin, 


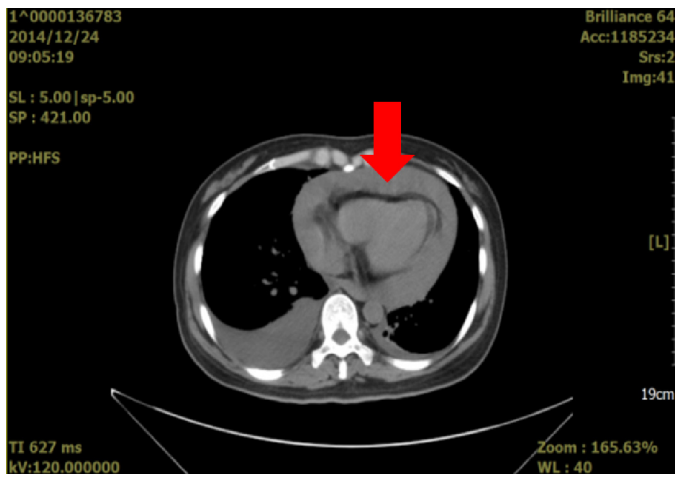

Figure I Computed tomography scan obtained on December 24, 2014 (7 days before treatment).

Notes: Scan demonstrates a mass in the upper lobe of the left lung, multiple enlarged lymph nodes in the mediastinum, and pleural effusion; arrow indicates abundant pericardial effusion around the pericardium.

and bevacizumab, and exhibited a partial response, as determined by using the Response Evaluation Criteria in Solid Tumors. She then received erlotinib irregularly.

When the patient was readmitted in December 2014, we drained approximately $1,100 \mathrm{~mL}$ of hemorrhagic effusion by using a catheter through a pericardial puncture. Bevacizumab was perfused into the pericardium at a dose of $15 \mathrm{mg} /$ $\mathrm{m}^{2}$. After surgery, the symptoms of chest distress and asthma were gradually alleviated, and ultrasonography revealed only a small amount of MPCE. Fifteen days after surgery, the patient received $50 \mathrm{~Gy} / 25$ fr of radiotherapy to the chest. She was discharged from the hospital 5 days later, and continued to receive pemetrexed monthly. Clinical examinations were performed every month, including a physical examination, determination of serum carcinoembryonic antigen concentration, and chest $\mathrm{CT}$ or ultrasonography. For 4 months after the bevacizumab treatment, the patient did not experience chest distress, suppression, palpitation, or any other symptoms. CT scans (Figures 2 and 3) and an

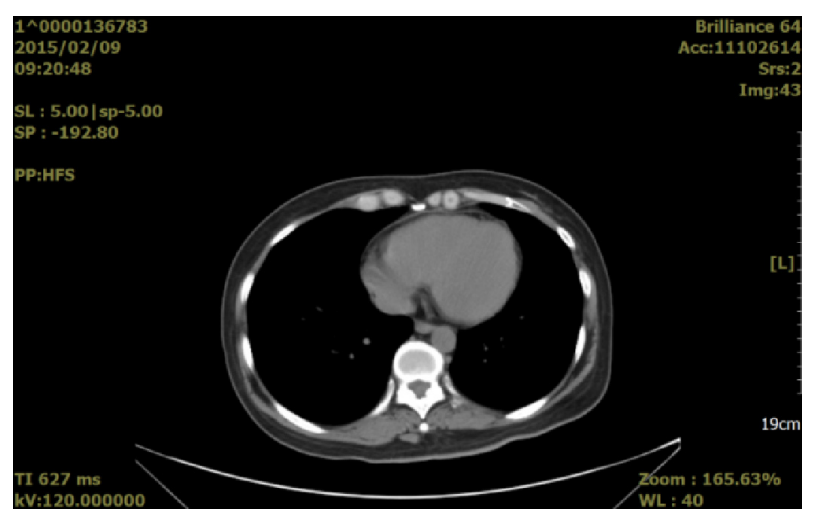

Figure 2 Pericardial effusion-volume changes seen on a computed tomography image obtained on February 9, 2015 ( 2 months after bevacizumab treatment).

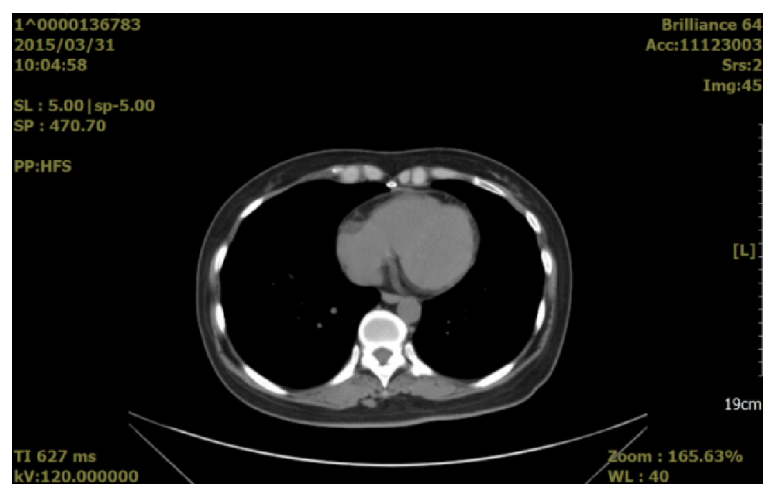

Figure 3 Pericardial effusion-volume changes seen on a computed tomography scan obtained on March 3I, 2015 (4 months after bevacizumab treatment).

ultrasonogram (Figure 4) did not show effusion in any of the three follow-up examinations. The study was approved by the institutional review board of Radiation Oncology, Shandong Cancer Hospital and Institute. The patient provided written informed consent.

\section{Discussion}

MPCE describes the abnormal infiltration of fluid into the pericardium induced by tumor metastasis. ${ }^{3}$ As a severe complication of advanced lung cancer, MPCE can cause hepatosplenomegaly, acute and chronic pericardial tamponade, and respiration deterioration. Severe symptoms of MPCE include respiratory distress, heart failure, and shock, thereby decreasing overall survival and patient quality of life, possibly resulting in death. ${ }^{4}$ Thus far, treatments available for MPCE include punctured drainage, use of diuretics, pericardial perfusion of antitumor agents, and treatment of the primary tumor. The most common method for treatment of MPCE is pericardial injection of cytotoxic drugs, which can directly kill tumor cells and eliminate effusion. ${ }^{5}$ Cisplatin is the most common agent for treating malignant effusion

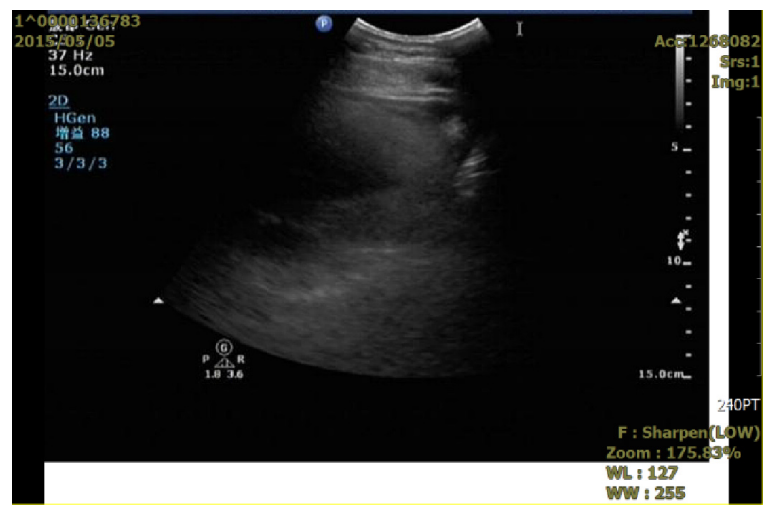

Figure 4 Pericardial effusion-volume changes seen on an ultrasonogram obtained on May 5, 2015 (5 months after bevacizumab treatment). 
because of its high patient response rate; however, it is associated with common chemotherapeutic adverse events. ${ }^{6}$ Chen et al reported a complete remission rate of $71.4 \%$ in non-small-cell lung cancer (NSCLC) patients treated with etoposide and cisplatin by pericardial perfusion to alleviate MPCE. ${ }^{7}$ However, the authors also reported MPCE recurrence and gastrointestinal and hematological toxicities in most patients. Moreover, pericardial perfusion of IL-2 exhibited relatively poor efficiency compared with traditional chemotherapy drugs, and induced fever in $73.3 \%$ of lung cancer patients with MPCE. ${ }^{8}$ These studies support the idea that routine methods for MPCE treatment are substandard, owing to the occurrence of severe adverse events. In addition, these treatments are associated with poor efficiency and high recurrence rates. Therefore, there is an urgent need for an effective treatment for MPCE to improve patient prognosis and quality of life.

Tumor metastases to serous cavities, such as the pleural cavity, abdominal cavity, or pericardial cavity, greatly elevate local concentrations of VEGF. 9,10 VEGF, the most effective angiogenic growth factor, not only promotes tumor growth and tumor angiogenesis but also increases capillary permeability, leading to fluid effusion into the serous cavity. ${ }^{11}$ Bevacizumab, a humanized monoclonal antibody against the VEGF receptor, can specifically block VEGF, inhibit tumor angiogenesis, normalize tumor vessels, and reduce capillary permeability. ${ }^{12}$ Ribeiro et $\mathrm{al}^{13}$ induced pleural effusion in 112 rabbits through intrapleural injections of either talc or silver nitrate, and administered intravenous injections of bevacizumab. They found that animals pretreated with bevacizumab exhibited a significant reduction in pleural effusion, macroscopic pleural adhesion scores, and vascular permeability. Luo et al examined the expression of VEGF in 13 mice with ascites tumors, and found that the concentration of active VEGF significantly increased $(6-850 \mathrm{ng} / \mathrm{mL})$ in the ascites fluids of all 13 mice. ${ }^{14}$ Moreover, VEGF concentrations were higher in tumors with a sarcoma and carcinoma origin $(430.4 \pm 234.2 \mathrm{ng} / \mathrm{mL})$ than in those with a lymphoma and hematological origin $(19.2 \pm 10.45 \mathrm{ng} / \mathrm{mL}) .{ }^{14}$ The authors concluded that VEGF plays a principal role in the formation of ascites tumors and differentially influences tumors of various origins.

Recent clinical studies have suggested that intraperitoneal perfusion of bevacizumab in combination with chemotherapeutic drugs results in higher efficiency and lower occurrence of adverse events compared with perfusion of cytotoxic chemotherapeutic drugs alone. El-Shami et al reported that an 82-year-old woman with refractory ovarian cancer and malignant ascites received an intraperitoneal perfusion of bevacizumab, after which her ascites symptoms were alleviated without any adverse reaction. ${ }^{15}$ Numnum et al reported symptomatic relief of ascites without grade 3/4 toxicity in four patients after intraperitoneal bevacizumab treatment. They concluded that bevacizumab might be a viable palliative option in patients with end-stage ovarian carcinoma who have symptomatic ascites. ${ }^{16}$ In a study by Du et $\mathrm{al}^{17} 72$ NSCLC patients with malignant pleural effusion were randomly assigned to two groups. One group received intrapleural bevacizumab combined with cisplatin, and the other group received intrapleural cisplatin alone. The curative efficacy in the combined group was significantly higher than that in the cisplatin group ( $83.3 \%$ vs $50 \%$, respectively; $P<0.05)$. After treatment, the pleural effusion levels of VEGF were significantly lower in the bevacizumab group compared to the cisplatin group $(P<0.01)$.

Both preclinical and clinical studies support intracavitary perfusion of bevacizumab as an efficient treatment for malignant effusion of the serous cavity. However, to date there are no data on the use of intracavitary perfusion of bevacizumab to treat MPCE. We report the intracavitary perfusion of bevacizumab and the subsequent clinical response in a patient with lung adenocarcinoma. The patient had received multiline treatments, and yet her disease showed rapid progression. She presented with significant PCE, and required urgent treatment to alleviate her symptoms. However, the patient's performance status was extremely poor, resulting in her inability to receive cytotoxic drug therapy. Based on a thorough review of the literature, which indicated that bevacizumab could play a predominant role in the treatment of malignant effusion, we treated the patient's MPCE with bevacizumab by pericardial perfusion. Soon after the treatment, the patient's clinical symptoms disappeared and her MPCE went into complete remission, as determined on ultrasonography. The patient sustained this response, with the MPCE and malignant pleural effusion remaining in remission for 4 months. Meanwhile, local radiotherapy and systemic chemotherapy were administered to enhance the effect. In this case, pericardial perfusion of bevacizumab efficiently alleviated patient symptoms and MPCE without any adverse events, suggesting that this treatment could control the symptoms of MPCE, improve patient quality of life, and prolong survival.

\section{Conclusion}

This case report indicated that pericardial perfusion of bevacizumab inhibited PCE and relieved patient symptoms. 
Drainage through a pericardial puncture and subsequent perfusion of bevacizumab may be a valuable method to control MPCE in patients with NSCLC. However, as a single case report, this study has several limitations. It is necessary to evaluate additional cases to validate these findings. Moreover, clinical studies are needed to investigate the effective dose of bevacizumab as well as the appropriate course of treatment. Finally, studies evaluating both the therapeutic effects and the adverse events of bevacizumab in combination with chemotherapeutic drugs are necessary to characterize fully the use of this treatment.

\section{Acknowledgment}

This work was supported by the Wu Jieping Medical Foundation (320.6750.14021).

\section{Disclosure}

The authors report no conflicts of interest in this work.

\section{References}

1. McAllister HA Jr, Hall RJ, Cooley DA. Tumors of the heart and pericardium. Curr Probl Cardiol. 1999;24(2):57-116.

2. Jama GM, Scarci M, Bowden J, Marciniak SJ. Palliative treatment for symptomatic malignant pericardial effusion. Interact Cardiovasc Thorac Surg. 2014;19(6):1019-1026.

3. Klatt EC, Heitz DR. Cardiac metastases. Cancer. 1990;65(6):1456-1459.

4. Skhvatsabaja LV. Secondary malignant lesions of the heart and pericardium in neoplastic disease. Oncology. 1986;43(2):103-106.

5. Martinoni A, Cipolla CM, Civelli M, et al. Intrapericardial treatment of neoplastic pericardial effusion. Herz. 2000;25(8):787-793.
6. Oida T, Mimatsu K, Kano H, et al. Pericardiocentesis with cisplatin for malignant pericardial effusion and tamponade. World J Gastroenterol. 2010;16(6):740-744.

7. Chen LK, Xu GC, Liang Y, Yang QY, Zhang LN. [Intrapericardial infusion of etoposide and cisplatin in treating malignant pericardial effusion of non-small cell lung cancer]. Ai Zheng. 2006;25(4):505-508. Chinese.

8. Millaire A, Wurtz A, Brullard B, et al. [Value of pericardioscopy in patients with pericardial effusion. A propos of 20 patients]. Arch Mal Coeur Vaiss. 1988;81(9):1071-1076. French.

9. Zhou WB, Bai M, Jin Y. Diagnostic value of vascular endothelial growth factor and endostatin in malignant pleural effusions. Int J Tuberc Lung Dis. 2009;13(3):381-386.

10. Nagy JA, Dvorak AM, Dvorak HF. Vascular hyperpermeability, angiogenesis, and stroma generation. Cold Spring Harb Perspect Med. 2012;2(2):a006544.

11. Sriram KB, Relan V, Clarke BE, et al. Diagnostic molecular biomarkers for malignant pleural effusions. Future Oncol. 2011;7(6):737-752.

12. Midgley R, Kerr D. Bevacizumab - current status and future directions. Ann Oncol. 2005;16(7):999-1004.

13. Ribeiro SC, Vargas FS, Antonangelo L, et al. Monoclonal anti-vascular endothelial growth factor antibody reduces fluid volume in an experimental model of inflammatory pleural effusion. Respirology. 2009;14(8): $1188-1193$.

14. Luo JC, Yamaguchi S, Shinkai A, Shitara K, Shibuya M. Significant expression of vascular endothelial growth factor/vascular permeability factor in mouse ascites tumors. Cancer Res. 1998;58(12):2652-2660.

15. El-Shami K, Elsaid A, El-Kerm Y. Open-label safety and efficacy pilot trial of intraperitoneal bevacizumab as palliative treatment in refractory malignant ascites. J Clin Oncol. 2007;25(18 Suppl):9043.

16. Numnum TM, Rocconi RP, Whitworth J, Barnes MN. The use of bevacizumab to palliate symptomatic ascites in patients with refractory ovarian carcinoma. Gynecol Oncol. 2006;102(3):425-428.

17. Du N, Li X, Li F, et al. Intrapleural combination therapy with bevacizumab and cisplatin for non-small cell lung cancer-mediated malignant pleural effusion. Oncol Rep. 2013;29(6):2332-2340.
OncoTargets and Therapy

\section{Publish your work in this journal}

OncoTargets and Therapy is an international, peer-reviewed, open access journal focusing on the pathological basis of all cancers, potential targets for therapy and treatment protocols employed to improve the management of cancer patients. The journal also focuses on the impact of management programs and new therapeutic agents and protocols on

\section{Dovepress}

patient perspectives such as quality of life, adherence and satisfaction. The manuscript management system is completely online and includes a very quick and fair peer-review system, which is all easy to use. Visit http://www.dovepress.com/testimonials.php to read real quotes from published authors. 